\title{
An Electrical Model to U-Slot Patch Antenna with Circular Polarization
}

\author{
Guesmi Chaouki \\ Department of Physics, FST \\ Unit of Research in High Frequency Electronic \\ Circuits and Systems \\ Necibi Omrane \\ Department of Physics, FST \\ Unit of Research in High Frequency Electronic \\ Circuits and Systems
}

\author{
Ghnimi Said \\ Department of Physics, FST \\ Unit of Research in High Frequency Electronic \\ Circuits and Systems \\ Gharsallah Ali \\ Department of Physics, FST \\ Unit of Research in High Frequency Electronic \\ Circuits and Systems
}

\begin{abstract}
The microstrip antenna is one of the best antenna structures, due to its low cost and compact design. In this paper, a coaxial feed circularly polarized square patch antenna is designed using the U-slot. The proposed antenna is suited for the RFID readers in the SHF band. This structure of antenna of FR4 substrate (dielectric constant $=3.5$ ), is capable to cover the range of frequency of 2.4 to $2.5 \mathrm{GHz}$. The size of patch is $25 * 25$ $\mathrm{mm}^{2}$. An equivalent electrical model of this antenna was proposed and simulated by the ADS software. The simulated gain is $4.189 \mathrm{dBi}$ and $S_{11}$ bandwidth is about $100 \mathrm{MHz}$. Analysis and modeling of the proposed antenna was carried out using the CST and HFSS simulator based on the finite element method. The simulation results obtained are presented and discussed.
\end{abstract}

Keywords-RFID; circularly polarization; U-slot antenna; RFID reader antenna; Electrical model

\section{INTRODUCTION}

Previously, the circular polarization was created by feeding the antenna to different locations and with a $90^{\circ}$ phase shift. At that time, the feeding was made directly (without slot) using a coaxial cable or micro-strip line. With the feeding in one place, circular polarization is induced either by making the antenna slightly rectangular (instead of square). Either by cutting two of its corners or by making a diagonal opening in its metallization. These three topologies have been studied by Sharma et al. [1].

The choice in this work stopped on the antenna with truncated corners. In addition to maintaining symmetry at the diagonal, this configuration is easier to conceive since it has a degree of freedom of less than the opening in the metallization. The latter can vary in length and width while the truncation is symmetrical. According to Sharma [1], the antenna with truncated corners provides the lowest axial ratio. But has a slightly smaller bandwidth (axial ratio) than the other topologies [2]-[4].

Many techniques have already been applied to the design of broadband antennas. For example, an insulated slot in a patch, the addition of different slot shapes at the radiating element, such as the L-shaped slot, a T-shaped slot, an H-shaped slot and the fractals slot have also been reported for large bands [5]-[9]. Among these techniques, a U-shaped slot will be used on the patch of the coaxially fed square patch antenna.

These types of antennas also have broad applications in long range and wireless identification or communication systems, such as RFID which is one of the new identification techniques and where the size of the system depends essentially on the size of the antenna [10]. In order to analyze this structure, a new electrical model is developed and compared to a physical patch.

Many calculation methods are adopted to resolve the maxwell equations and then analyse the performance of the antennas. Among these, three of them are broadly used in simulation software:

1) The method of moments (MoM) is used among others in ADS software.

2) The method of finite integral (FIT) is used in the software CST Microwave Studio [11], [12].

3) The Finite Element Method (FEM) is used in the software HFSS [11].

In this work, the method of moments will be adopted to analyze the performance of the electrical model and the results will be compared with those obtained by the Finite Integral and the Finite Element Method.

\section{The Approaches to Get the EleCtriCAL MOdEL OF THE SQUARE PATCH ANTENNA}

The patch antenna can be modeled simply by a parallel or serial RLC circuit. To calculate its characteristics, the study based on the RLC circuit is the most used. In the next step, the parameters of the RLC circuit in the equivalent electrical model of the patch antenna will be calculated. The parameters of the proposed model are determined using the same solution of Nasimuddin and A. K. Verma [17]. 


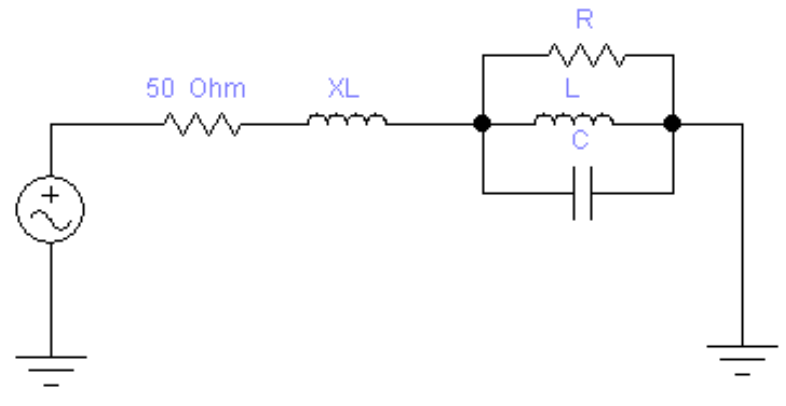

Fig. 1. Electrical model of square patch

The input impedance of a square patch excited by a coaxial cable is given in [18] by equation (1):

$$
\begin{gathered}
Z_{\text {in }}=R+j X \\
Z_{\text {in }}=\frac{R}{1+Q_{T}^{2}\left(\frac{f}{f_{r}}-\frac{f_{r}}{f}\right)^{2}}+j\left(X_{L}-\frac{R Q_{T}\left(\frac{f}{f_{r}}-\frac{f_{r}}{f}\right)}{1+Q_{T}^{2}\left(\frac{f}{f_{r}}-\frac{f_{r}}{f}\right)^{2}}\right)
\end{gathered}
$$

The resonant resistance $\mathrm{R}$ of our parallel circuit RLC is given in [18] by equation (2):

$$
R=\frac{Q_{T} h}{\pi f_{r} \varepsilon_{d y n} \varepsilon_{0} A} \cos ^{2}\left(\frac{\pi X_{0}}{a}\right)
$$

$\mathrm{f}_{\mathrm{r}}$ : resonant frequency.

$\mathrm{X}_{0}$ : the distance of the feed point from the edge of the patch.

h: thickness of dielectric.

A : air of the square patch

$\mathrm{a}:$ length of the edge

The total Quality factor $Q_{T}$ is calculating in [18] by equation (3):

$$
Q_{T}=\frac{1}{\frac{1}{Q_{R}}+\frac{1}{Q_{C}}+\frac{1}{Q_{D}}}
$$

$Q_{R}$ is the radiation quality factor, $Q_{D}$ is the losses in the dielectric and $\mathrm{Q}_{\mathrm{C}}$ is losses in conductor.

$$
\begin{gathered}
Q_{R}=\frac{C_{0} \sqrt{\varepsilon_{d y n}}}{4 f_{r} h} \\
Q_{C}=\frac{0.786 \sqrt{f_{r} Z_{a} a} h}{P_{a}}
\end{gathered}
$$

$$
Q_{D}=\frac{1}{T_{g} \delta}
$$

The impedance of a microstrip line filled with air " $\mathrm{Za"}$ " is given by equation (7):

$$
\begin{aligned}
& Z_{a}(a)=\frac{60 \pi}{\sqrt{\varepsilon_{r}}}\left(\begin{array}{l}
\frac{a}{2 h}+0.441+0.082\left(\frac{\varepsilon_{r}-1}{\varepsilon_{r}^{2}}\right) \\
+\frac{\varepsilon_{r}+1}{2 \pi \varepsilon_{r}}\left(1.451+L_{n}\left(\frac{a}{2 h}+0.94\right)\right)
\end{array}\right) \\
& Z_{a 0}(a)=Z_{a}\left(a, \varepsilon_{r}=1\right) \\
& P_{a}=\frac{2 \pi\left(\frac{a}{h}+\frac{\frac{a}{\pi h}}{\frac{a}{2 h}+0.94}\right)\left(1+\frac{a}{h}\right)}{\left(\frac{a}{h}+\frac{2}{\pi} L_{n}\left(2 \pi \exp \left(\frac{a}{2 h}+0.94\right)\right)^{2}\right.} ; \frac{a}{h} \geq 2
\end{aligned}
$$

The dynamic permittivity $\varepsilon_{\text {dyn }}$ is calculated by (9):

$$
\begin{gathered}
\varepsilon_{d y n}=\frac{C_{d y n}(\varepsilon)}{C_{d y n}\left(\varepsilon_{0}\right)} \\
C_{d y n}(\varepsilon)=\frac{\varepsilon_{0} \varepsilon_{r} A}{h y_{n} y_{m}}+\frac{1}{2 y_{n}}\left(\frac{\varepsilon_{r e f f}\left(a, h, \varepsilon_{r}\right)}{C_{0} Z\left(a, h, \varepsilon_{r}=1\right)}-\frac{\varepsilon_{0} \varepsilon_{r} A}{h}\right) \\
y_{j}=\left\{\begin{array}{l}
1, j=0 \\
2, j \neq 0
\end{array}\right. \\
Z\left(a, h, \varepsilon_{r}=1\right)=\frac{377}{2 \pi} L_{n}\left(\frac{f \frac{a}{h}}{\frac{a}{h}}+\sqrt{1+\left(\frac{a}{2}\right)^{2}}\right) \\
f\left(\frac{a}{h}\right)=6+(2 \pi-6) \exp \left(-\left(\frac{30.666}{\frac{a}{h}}\right)^{0.758}\right)
\end{gathered}
$$

The formula of $\mathrm{C}_{\mathrm{dyn}}$ is used to determine the capacity $\mathrm{C}$ and to determine the inductance $\mathrm{L}$ we use the following equations:

$$
\begin{gathered}
w_{\text {res }}=2 \pi f_{r} \\
w_{\text {res }}=\frac{1}{\sqrt{L C}} \Rightarrow L=\frac{1}{w_{\text {res }}^{2} C}
\end{gathered}
$$

Equation (15) allows us to calculate the inductive reactance of coax, taking $\mathrm{d} 0$ the diameter of the probe:

$$
X_{L}=\frac{377 f h}{C_{0}} L_{n}\left(\frac{C_{0}}{\pi f d_{0} \sqrt{\varepsilon_{0}}}\right)
$$




\section{ANTENNA STRUCTURE AND DESIGN}

The geometry of the proposed antenna is shown in figure 1 . The square patch antenna was truncated to create a circular polarization (CP). The proposed antenna is printed on a FR-4 substrate of relative permittivity $\varepsilon_{\mathrm{r}}=3.5$ and thickness $\mathrm{h}=3.2$ $\mathrm{mm}$ and fed by a coaxial cable. Many studies have practiced this mode of feeding [13]-[15]. The substrate is stacked with two layers of FR-4 to allow for greater bandwidth, higher gain and efficiency radiation. In order to increase the impedance and the bandwidth of S11, the truncated square patch antenna has been loaded by a U-slot which introduces a capacitance making it possible to eliminate the inductance due to the vertical feed probe.

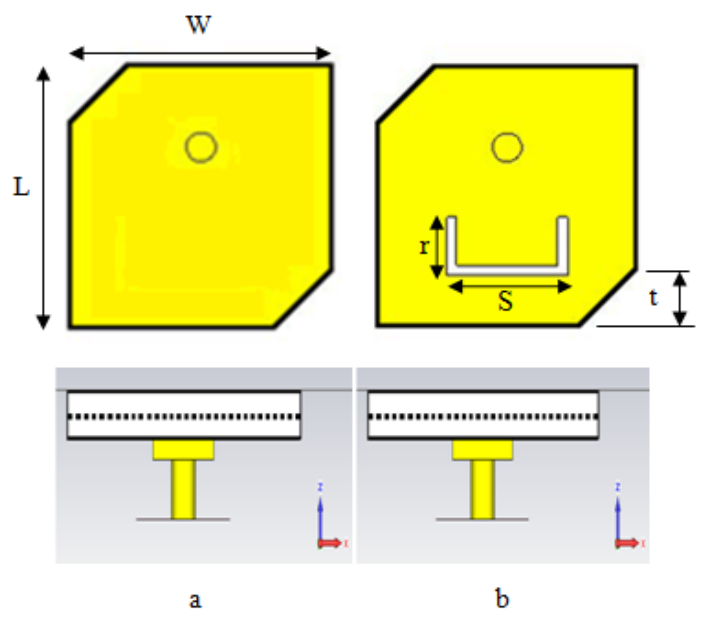

Fig. 2. Design for U-slot Patch Antenna (a): without the slot, (b): with the slot

TABLE. I. OPTIMIZED PARAMETERS OF THE PROPOSED RFID U-SLOT PATCH ANTENNA

\begin{tabular}{|l|l|l|l|l|l|l|l|}
\hline Parameters & t & r & s & W & L & $\varepsilon_{\mathrm{r}}$ & h \\
\hline Value $(\mathbf{m m})$ & 5 & 6 & 12 & 25 & 25 & 3.5 & 3.2 \\
\hline
\end{tabular}

The truncations made on the square antenna make this element the most difficult to conceive. When taken individually, it can be modeled by a disturbed cavity. The latter will induce a resonant mode perpendicular to that existing without the truncations. To obtain a circular polarization, these modes must have the same amplitude and be out of phase by $90^{\circ}$. The study of a truncated cavity was carried out by Haneishi et al., [16] who set up an equivalent circuit In the case of an antenna fed by a coaxial cable, the resonance frequency of the orthogonal modes $\left(f_{r l}\right.$ and $f_{r 2}$ ) is calculated as a function of the surface of the non-truncated antenna $(\mathrm{T})$ and the total truncated surface $(\Delta \mathrm{t})$ The relation between all these parameters is given by [16]

$$
\left|\frac{\Delta_{t}}{T}\right| Q_{0}=\left|\frac{\Delta_{t}}{T}\right| \frac{f_{0}}{\Delta_{f}}=\left|\frac{\Delta_{t}}{T}\right| \frac{f_{0}}{f_{r 1}-f_{r 2}}=\frac{1}{2}
$$

$Q_{0}$ is the quality factor of the cavity and $f_{0}$ is the resonance frequency of the undisturbed antenna.

\section{RESULTS AND DISCUSSION}

Characteristics of the proposed patch antenna were simulated in this section using the CST software. The simulated curves of return loss as a function of the frequency by varying the section " $\mathrm{t}$ " of the proposed antenna are shown in figure 3 . It is noted that the proposed antenna with $\mathrm{t}=5 \mathrm{~mm}$ gives the best return loss to the antenna desired frequency. Therefore, we fixed $\mathrm{t}=5 \mathrm{~mm}$ and cut a U-shaped slot in the radiating element of the proposed antenna to observe the variations of the return loss. The simulated results are presented in figure4. From this figure it is easy to notice that the desired frequency at $2.45 \mathrm{GHz}$ of the proposed antenna is obtained by the slotted structure.

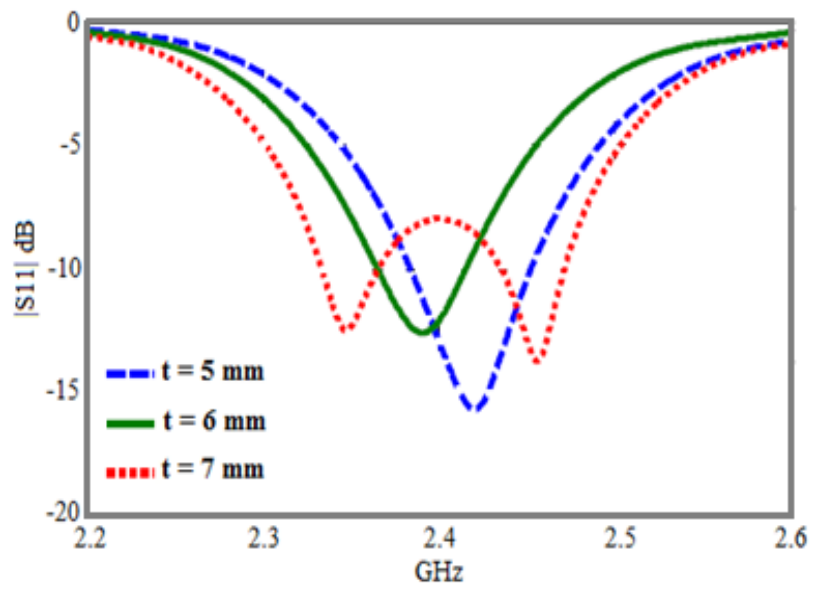

Fig. 3. Simulated return loss of square patch antenna with varieties value of (t)

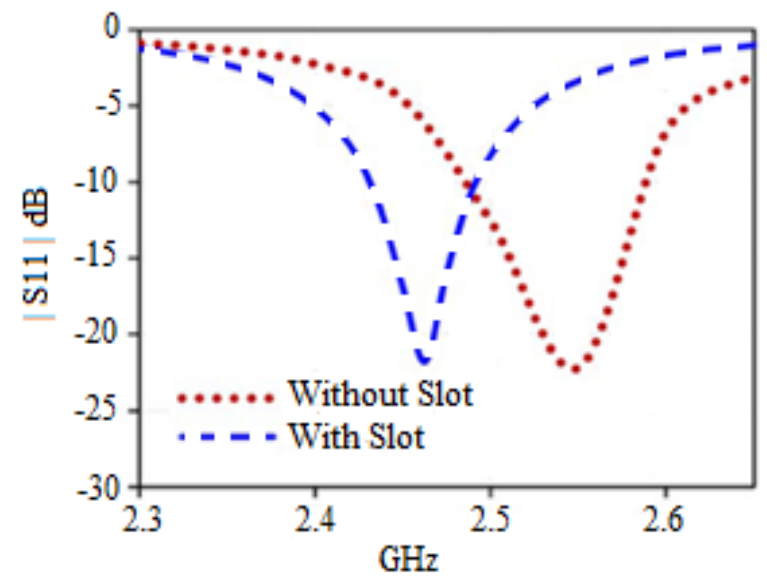

Fig. 4. Simulated return loss for the proposed antenna with and without the slot (with CST)

Figure 5 shows the response of the reflection coefficient of the proposed antenna obtained from the simulation CST and HFSS with respect to the calculated response of the equivalent circuit model. 


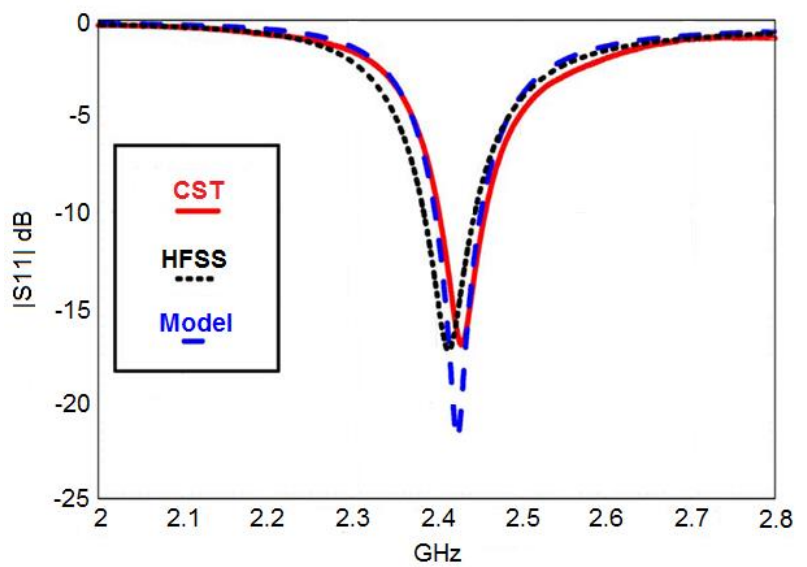

Fig. 5. Simulated return loss for the proposed antenna

Figure 5 shows the reflection coefficient S11 of the proposed antenna obtained from CST and HFSS relative to that obtained from the equivalent circuit using ADS. In Table II the detailed characteristics of these responses are listed. It can be noted from Table II that the requirement of the SHF band 2.45 $\mathrm{GHz}$ is satisfied. The results simulated by CST and HFSS agree with that obtained from ADS with some slight differences. This difference is due to the fact that the structure simulator in the CST and HFSS software accounts for all the coupling effects in the simulated antenna physical structure whereas in the equivalent circuit model only the individual elements are taken into account without taking into account the coupling between them.

TABLE. II. COMPARISON OF THE BAND CHARACTERISTICS OBTAINED

\begin{tabular}{|l|l|l|l|l|l|}
\hline & $\begin{array}{l}\text { S11 } \\
(\mathbf{d B})\end{array}$ & $\begin{array}{l}\text { Start freq } \\
(\mathbf{G H z})\end{array}$ & $\begin{array}{l}\text { End freq } \\
(\mathbf{G H z})\end{array}$ & $\begin{array}{l}\text { Center } \\
\text { freq }(\mathbf{G H z})\end{array}$ & $\begin{array}{l}\text { BW } \\
(\mathbf{G H z})\end{array}$ \\
\hline CST & -18 & 2.40 & 2.47 & 2.45 & 0.07 \\
\hline HFSS & -18 & 2.38 & 2.44 & 2.41 & 0.06 \\
\hline ADS & -22 & 2.39 & 2.46 & 2.43 & 0.07 \\
\hline
\end{tabular}

Figure 6 shows the axial ratio as a function of the frequency. We chose the criterion RA $<3 \mathrm{~dB}$ to measure the bandwidth. Note that the bandwidth is also very low. In effect, its value is only $0.30 \%$. This low value comes from the use of truncated corners. Sharma [1] showed that this type of antenna could not provide a large bandwidth at the axial ratio.

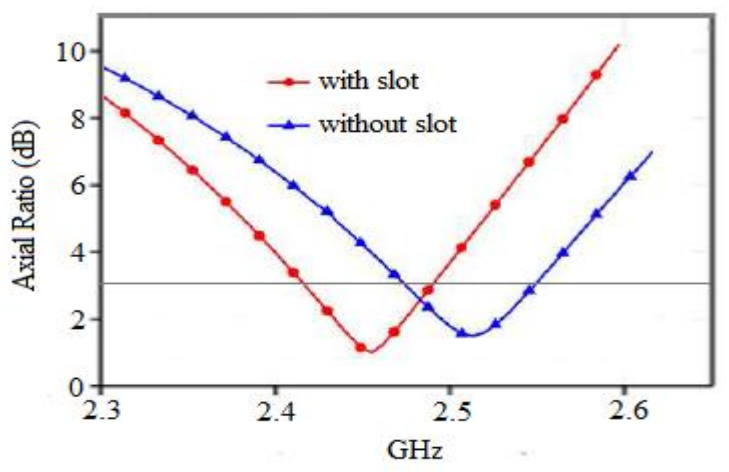

Fig. 6. Simulated axial ratio for the proposed antenna
Figure 7 shows the 3D radiation pattern of the proposed antenna for the resonant frequency $2.45 \mathrm{GHz}$. We note that for this frequency, we have a directional diagram, the efficiency of the radiation which equals $96.7 \%$ and the total efficiency is $93.3 \%$. The gain obtained is $4.29 \mathrm{~dB}$. The HPBW value is 92.0 Deg.

The linear and nonlinear gain of theta / phi $0^{\circ}$ in polar form are represented respectively by figure 8 .

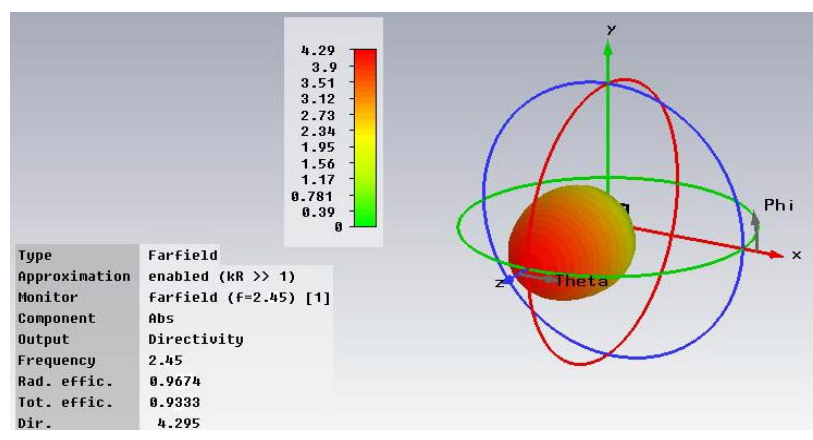

Fig. 7. 3D Farfield for U-slot antenna

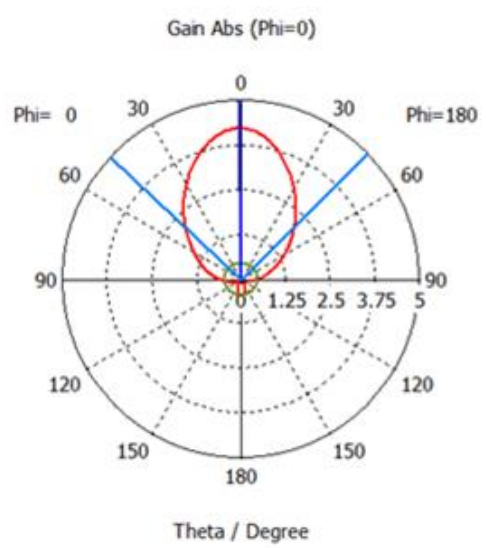

farfield $(f=2.45)[1]$

(a)

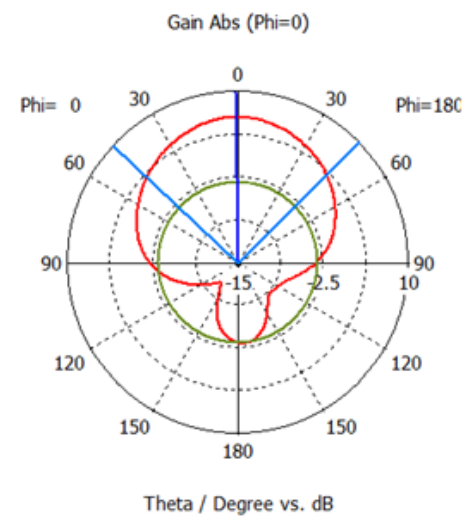

Frequency $=2.45$

Main lobe magntude $=4.18 \mathrm{~dB}$ Man lobe drection $=1.0 \mathrm{deg}$. Angular width $(3 \mathrm{~dB})=92.0 \mathrm{deg}$. Side lobe level $=-8.9 \mathrm{~dB}$

(b)

Frequency $=2.45$ Main lobe magnitude $=5.8 \mathrm{~dB}$ Main lobe direction $=1.0 \mathrm{deg}$. Angular width $(3 \mathrm{~dB})=92.0 \mathrm{deg}$. Side lobe level $=-8.9 \mathrm{~dB}$

Fig. 8. The polar pattern: (a) Linear and (b) Non-linear

Figure 9 shows the propagation of current from the coaxial feed in the patch. Indeed, by truncating the patch antenna, it generates a circular polarization $(\mathrm{CP})$. Note that when the upper right part and the lower left part of the resulting truncated refers to the right circular polarization, RHCP. The 
antenna can work with truncated LHCP at another diagonal axis.

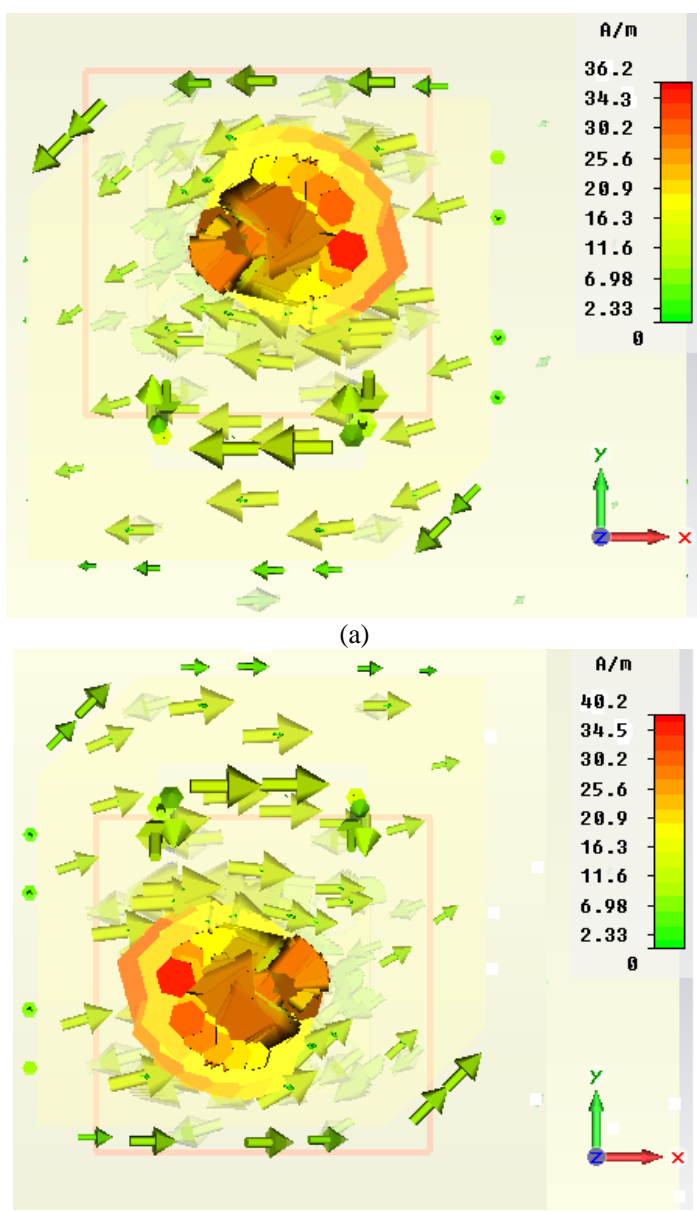

(b)

Fig. 9. Surface current at U-slot Patch Antenna, (a): LHCP and (b): RHCP

\section{CONCLUSIONS}

Evaluation and study of antennas is based on their properties and characteristics. The latter vary from one application to another. According to the requirements defined by the application, the antenna design method can be chosen. Thus, each method has its advantages in well-defined cases. For our application, we chose the method of electrical modeling for the analysis of square patch antenna structures referred to RFID readers.

The proposed antenna is circularly polarized using the truncation method. Indeed, the LHCP and RHCP polarization depend on the corners of the diagonal axis which are truncated. This truncated square patch antenna with U-shaped slot has been designed to achieve high bandwidth, high radiation efficiency and resonant frequency equal to $2.35 \mathrm{GHz}$.

The modeling method that we have applied in this work has several advantages. In fact, it allows to simulate structures in a simple, fast and efficient way. In addition, once the model is built, we can track changes in antenna parameters based on changes in antenna geometry, position and nature of excitation.
So we can integrate these antenna structures easily into RFID systems.

\section{REFERENCES}

[1] X. Ye, M. He, P. Zhou and H. Sun, "A Compact Single-Feed Circularly Polarized Microstrip Antenna with Symmetric and Wide-Beamwidth Radiation Pattern." International Journal of Antennas and Propagation Volume 1, 2013.

[2] M. F. Jamlos, M. R. Kamarudin, M.A. Jamlos and M. Jusoh, " A novel reconfigurable quadratic antenna for wimax and 4G systems" MICROWAVE AND OPTICAL TECHNOLOGY LETTERS, Pages: 416-421, Volume 54, Issue 2, February 2012

[3] M. Jusoh, M. F. Jamlos and M. R. Kamarudin, "A compact dual bevel planar monopole antenna with lumped element for ultra-high frequency/very high frequency application", MICROWAVE AND OPTICAL TECHNOLOGY LETTERS, Pages: 156-160Volume 54, Issue 1, January 2012

[4] M. Jusoh, M. F. Jamlos, M. R. B. Kamarudin, and M. F. b. A. Malek, "A MIMO antenna design challenges for UWB application, " Progress In Electromagnetics Research B, Vol. 36, 357-371, 2012.

[5] W. LUI, C. CHENG and H. ZHU, "Improved frequency notched ultra wideband slot antenna using square ring resonator", IEEE Trans. Antennas Propag., vol 55, No. 9, pp. 2445-2450, 2007.

[6] A. Farswan, A. K. Gautam, B. K. Kanaujia and K. Rambabu "Design of Koch Fractal Circularly Polarized Antenna for Handheld UHF RFID Reader Applications", IEEE Transactions on Antennas and Propagation, Vol 64, No 2, Feb. 2016.

[7] S. Lakrit, H. Ammor and J. Terhzaz, "Design of H-slot Patch Antenna for Ultra Wideband", European Journal of Scientific Research , Vol 106 No 2, pp:224-228, 2013.

[8] R. Zaker., C.Ghobadi and J.Nourinia, "Bandwidth enhancement of novel compact single and dual band-notched printed monopole antenna with a pair of L-shaped slots", IEEE Trans. Antennas Propag., vol 57, No. 12, pp. 3978-3983, 2009.

[9] N. Prombutr, P. Kirawanich, and P. Akkaraekthalin “ Bandwidth Enhancement of UWB Microstrip Antenna with a Modified Ground Plane" International Journal of Microwave Science and Technology, Volume11, 2009.

[10] K. Finkenzeller, "RFID HANDBOOK", Second Edition, 2003.

[11] Guy A. E. Vandenbosch and Alexander Vasylchenko, "A Practical Guide to 3D Electromagnetic Software Tools, " InTechEUROPE pages $504-541$,

[12] B. Bieda and P. Słobodzian, "Efficiency of the IE-MoM Approach in the Analysis of Dielectric Bodies Embedded in a Cavity," Microwave Radar and Wireless Communications (MIKON), 18th International Conference, 2010.

[13] A. Mak, C. R. Rowell, R. D. Murch, and M. Chi-Lun, "Compact multiband planar antenna for 2.4/3.5/5.2/5.8-GHz wireless applications," IEEE Antennas Wireless Propag. Lett., vol. 11, pp. 144-147, 2012.

[14] D. C. Chang, B. H. Zeng, and J. Liu, "CPW-fed circular fractal slot antenna design for dual-band applications," IEEE Trans. Antennas Propag., vol. 56, no. 12, pp. 3630-3637, Dec. 2008.

[15] B.D. Bala, M.K.A. Rahim, , N.A. Murad, M.F. Ismail and H.A. Majid, "Design and Analysis of Metamaterial Antenna Using Triangular Resonator," Microwave Conference Proceedings (APMC), Asia-Pacific, 2012.

[16] M. HANEISHI and S. YOSHIDA, "A Design Method of Circulary Polarized Rect angular Microstrip Antenna bp One-Point Feed." Electronics and Communications in Japan, 64B, 46-54. 1981.

[17] Nasimuddin and A. K. Verma Amir "Fast and accurate model for analysis of equilateral triangular patch antenna", Journal of Microwaves and Optoelectronics, Vol 3, pp 99-110, April 2004.

[18] S. Malisuwan, M. Charoenwattanaporn and Ut Goenchanart “ microstrip antenna Antenna for wireless lan by Appling modified smithchartrepresentation," International journal of the computer, the Internet and management, Vol 11, pp 34-44, 2003. 